\title{
Refractory Non-Hodgkin Lymphoma
}

National Cancer Institute

\section{Source}

National Cancer Institute. Refractory Non-Hodgkin Lymphoma. NCI Thesaurus. Code C8701.

A non-Hodgkin lymphoma which does not respond to treatment. 